\title{
Effect of Pseudo Random Noise (PRN) Spreading Sequence Generation of 3GPP Users' Codes on GPS Operation in Mobile Handset
}

\author{
Taher Al Sharabati
}

\begin{abstract}
In this paper, the effects of intersystem cross correlation of 3GPP user' codes to GPS satellites' codes will be demonstrated. The investigation and analysis are in the form of cross correlation between 3GPP users' codes and GPS satellites Pseudo Random Noise (PRN) sequences. The investigation and analysis will involve the similarities in generation and system architecture of both the 3GPP user' codes and GPS satellites' codes. The extent of intersystem interference will be displayed in the form of results for cross correlation, correlation coefficient, and signal to noise ratio. Recommendations will be made based on the results.
\end{abstract}

Index Terms - Gold Codes, 3GPP users' codes, GPS, interference, PRN sequences.

\section{INTRODUCTION}

$\mathrm{G}$ PS satellite coarse acquisition (C/A) codes are based on Gold codes and designed to have excellent intra cross correlation properties. User codes assigned to cellular customers in over the air (OTA) interface $3 \mathrm{G}$ and $4 \mathrm{G}$ networks are, also, based on Gold codes and designed to have excellent intra cross correlation properties. However, when both are operated in the same smart phone mobile handset, the user codes of the OTA will have cross correlation effects (in the form of intersystem interference) on the GPS C/A codes and therefore affecting the operation of the GPS receiver.

Because this kind of interference is particular and restricted to the mobile handset, no publications have been transmitted in the public domain. In fact, publications do not consider the mobile handset where this particular interference takes place, which makes this paper unique. In [1], a thorough investigation and study of the intra system Pseudo Random Noise (PRN) codes effects between Galileo E1 OS and GPS L1C were presented. However, these have different PRN code generation fundamentals where the Galileo E6 CS and E1 OS generation is based on random codes and GPS L1C generation is based on Weil codes. Even though they were different family of codes, the potential of intersystem interference was presented.

Manuscript received on June 22, 2016; revised October 19, 2016 and November 15, 2016.

Author is with the Al Ahliyya Amman University, Amman, Jordan (Email: t.sharabati@ammanu.edu.jo.)
Both GPS and 3GPP PRN codes generation, on the other hand, is based on Gold codes where their system architecture is identical. Conceptually, if [1] presented the potential of intra system interference between GPS and 3GPP PRN codes has higher likelihood and potential.

The novelty of this paper stems from the fact that no literature has presented the potential interference between GPS and 3GPP codes in the mobile handset. It presents the similarity in code generation between the GPS and 3GPP codes as well as the similarity in terms of their system architecture.

Other recent publications have treated interference to GPS from 3G carrier transmitters [2], [3]. However, the interference was based on power levels and data rates rather than code design generation and architecture of the $3 \mathrm{G}$ and GPS PRN codes.

The paper is organized as follows: Section II gives some brief background about some $3 \mathrm{G}$ basic technology especially the WCDMA and spreading codes. Section III will discuss Gold Code overview, background, system design aspects, and generation. It also demonstrates the similarity in system design and generation of GPS Gold Codes to those of 3GPP. Section IV presents the results based on simulations using Physical Layer characteristics and specification while Section $\mathrm{V}$ gives the conclusion.

\section{BACKGROUND}

In Wideband Code Division Multiple Access (WCDMA) technology, each user is assigned a unique, complex mixed sequence to encode its information-bearing signal. The receivers have to jostle the code of the user, un-jostle the received signal, and recapture the original information [4]. This application provides a process for complex pseudorandom noise sequence (PRN code) generation and a complex mix of an I/Q code multiplexed signal on a digital signal processor (DSP). The PRN codes in this application are produced for a WCDMA Universal Mobile Telecommunications Systems (UMTS) uplink (signal from handset or mobile station to base station) with reference to the third-generation partnership venture (3GPP) specifications [5].

WCDMA is a technology that has appeared as the most widely adopted third generation (3G) air interface. Traditionally, both of Gold code sequences and Pseudo 
Random Noise (PRN) sequences are used as spreading codes in WCDMA. However, improved performance by using these sequences is accomplished by increasing the length of the sequences [6]. WCDMA technology is the most widely used third generation system, which spreads over a wide bandwidth by combining both of the user data and of spreading sequence [7]. The different spreading codes with different spreading lengths are used to support high bit rate and reduction of the impact narrowband interference and inter-symbol interference (ISI) in WCDMA.

The main goal of the spreading codes is that they need to be orthogonal to each other. There are many types of spreading codes, but the most popular are Hadamard codes, PRN sequences, Gold sequences, and Walsh codes etc. PRN sequences are deterministically generated; however, they are nearly random sequences to an observer. The PRN sequences are usually obtained by using sequential logic circuits [8]. These binary sequences are shifted through the shift registers in retort to clock pulses, and the output of the different stages are logically combined and fed back as the input to the first stage. This shift register is called a linear PRN sequence generator [9].

\section{GPS AND 3GPP GOLD CODES SYSTEM ARCHITECTURE AND GENERATOR POLYNOMIAL DESIGN}

The nature of Gold codes design enabled GPS receivers to tune many of the signals at the same time without a moment's delay. Every GPS satellite propagates a "Coarse/Acquisition" (C/A) code. The C/A code is a recurrence, a pseudorandom digital code, that was selected from Gold codes classes.

Many characteristics and qualities characterize Gold codes. Because of these characteristics, they have been chosen as a pervasion concatenation for the GPS signals. From all these characteristics, the correlation attributes are the most essential features of C/A codes. The two substantial correlation features of the C/A codes can be illustrated as follows:

The first feature is, there should be almost zero cross correlation. That means almost there is no correlation between one code and other in all C/A codes. In other words, if we have two satellites $i$ and $k$ and their codes are $C^{i}$ and $C^{k}$, we can find the normalized cross correlation between them by the following equation:

$r_{i k}(m)=\frac{1}{r_{k k, \text { peak }}} \sum_{l=0}^{1022} C^{i}(l) C^{k}(l+m) \approx 0$

for all $m$ where $r_{k k, p e a k}$ is the peak magnitude as in (3).

The second feature is; almost no correlation except for zero lags. That means there is no correlation between one code and itself except at zero lag. This feature produces a simple way to know if there are two identical codes. We can find the normalized autocorrelation for satellite $k$ by the following equation:

$r_{k k}(m)=\frac{1}{r_{k k, p e a k}} \sum_{l=0}^{1022} C^{i}(l) C^{k}(l+m) \approx 0$

for $|m| \geq 1$.
Fig. 1 shows an example of both auto and cross correlation features of $\mathrm{C} / \mathrm{A}$ codes.
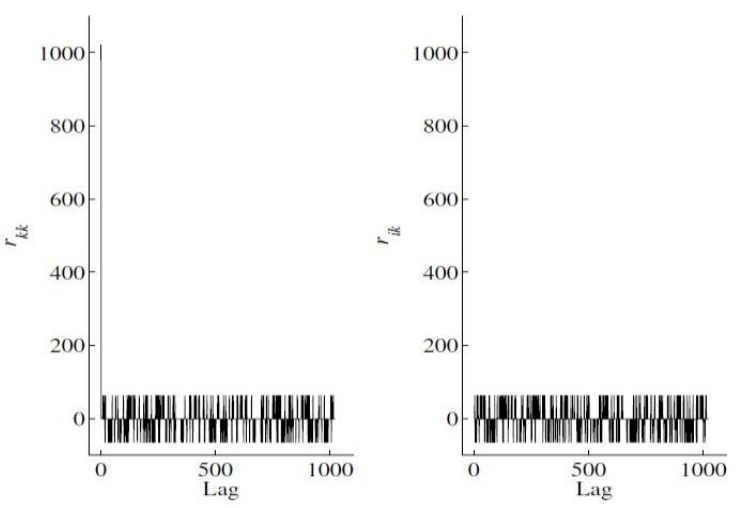

Fig.1. Correlation features of C/A codes. Left: Auto correlation $r_{k k}(m)$ of C/A code for PRN 1. Right: Cross correlation of the C/A codes for the PRNs 1 and 2 .

Fig. 1 illustrates high correlation at zero lag when correlating with the same $\mathrm{C} / \mathrm{A}$ code, and low correlation with a different $\mathrm{C} / \mathrm{A}$ code. The autocorrelation displayed in the left section of Fig. 1 has a peak of magnitude

$r_{k k, p e a k}=2^{n}-1=1023$

where $n$ indicates the number of states in the shift registers. In this situation $n=10$. The residual values satisfy the following difference,

$\left|r_{k k}\right| \leq 2^{(n+2) / 2}+1$

For $n=10$, we obtain, as shown in Fig. 1,

$\left|r_{k k}\right| \leq 65$

The cross correlation in the right section of Fig. 1 also satisfies the difference in equation (4).

In GPS, the movement of the satellite comparatively to the GPS receiver will produce a Doppler frequency shift. If we want to talk about fixed GPS receiver, the highest Doppler frequency shift for L1 frequency $(1575.42 \mathrm{MHz})$ is nearly \pm 5 $\mathrm{kHz}$. On the other hand, if we talk about GPS receiver traveling at a quick speed, it makes sense to consider the maximum Doppler shift to be nearly $\pm 10 \mathrm{kHz}$.

Doppler frequency shift is considered too small when compared to the minimal chip rate of the C/A code, which is $1.023 \mathrm{MHz}(1.023 \mathrm{Mc} / \mathrm{s})$. The C/A chip rate in turn is less than the L1 carrier frequency by about 1540 times. Based on that, the Doppler frequency value on C/A code is $3.2 \mathrm{~Hz}$ for a fixed GPS receiver and is $6.4 \mathrm{~Hz}$ for a fast moving GPS receiver. There is a possibility that an incorrect alignment will occur between the received signal and the locally generated codes [10].

The following analysis of pertain to both $3 \mathrm{G}$ and $4 \mathrm{G}$ since both of their spreading codes are based to some extent on Gold codes. 


\section{A. Structure of Gold Code}

The main fundamental feature of Gold codes is their pseudo random noise (PRN) nature, which is why they are also called PRN codes. PRN codes are a fundamental element of GPS and 3GPP. Codes are indeed a keystone of any system that relies on CDMA to separate different transmission channels, and thus to distinguish one satellite from the other [1].

The Gold code sequence generation is composed of two PRN sequences (Fig. 2) each of which uses a unique polynomial with the same length as LFSR, PRN sequence generation requires the shift operation and XOR to generate pseudo-random sequence. PRN sequence is specified by its polynomial. For example, a 6th degree polynomial is represented with the equation;

$x^{6}+x^{5}+x^{4}+x^{3}+x^{2}+x+1$

which results in 63 different possible codes of the same size, the length of each code with polynomial of degree $n$ is equal to $\left(2^{n}-1\right)$.

\section{B. Generating GPS Code}

The C/A codes are Gold codes with a period 1023 chips, transmitted at $1.023 \mathrm{Mbit} / \mathrm{s}$. we generate $\mathrm{C} / \mathrm{A}$ code by combining using exclusive or 2-bit streams generated by maximal period 10-stage LFSR. The C/A code generator contains two-shift register known as G1 and G2. These shift registers each have 10-cell generating sequence of length 1023. The two result in 1023 chips-long C/A code, only if the polynomial is able to generate code of maximum length. Every 1023rd period, the shift register are reset with all ones, making the code start over [11].

$G_{1}=x^{10}+x^{3}+1$

$G_{2}=x^{10}+x^{9}+x^{8}+x^{3}+x^{2}+1$.

$G_{2}{ }^{\prime}$ states $10,9,8,3$, and 2 are fed back to the input. In same way $G_{1}{ }^{\prime}$ states 10 and 3 are fed back to the input. To obtain different $\mathrm{C} / \mathrm{A}$ code, the output of two shift register is combined in a deterministic manner.

\section{Generating 3GPP Codes}

In 3GPP, Wideband Code Division Multiple Access (WCDMA) is used to allow multiple users transmit simultaneously within the same radio band channel. In order to enable frequency reuse, the network employs the spread spectrum technique [12]. To achieve that we can use Gold codes technique. In 3GPP, any two maximum length sequences can be combined, but two conditions must be satisfied to get to the real meaning of PRN:

1) Maximum length sequence should be the same length $2^{n}-1, n$ : size of LFSR

2) Absolute cross-correlation is less than $2^{(n+2) / 2}$.

In 3GPP, the polynomial determining the PRN sequence is called preferred polynomial. Determining the preferred polynomial depends on the number of users we want to serve Table 1 shows a short list of preferred polynomial pairing according to 3GPP. Each polynomial has an initial state vector that must be a binary number. The length of this vector should equal the degree of LFSR, and should contain at least one '1'. The sequence index range is $\left[-22^{n}-2\right]$.

TABLE I

Preferred Polynomial Pair SHORT List in 3GPP WHERE $\mathrm{n}=$ SIZE OF LFSR $\mathrm{N}=$ LENGTH OF THE $\mathrm{n}$ SEQUENCE [18 10665 0]=STRUCTURE OF POLYNOMIAL

\begin{tabular}{|c|c|c|c|c|}
\hline $\begin{array}{c}\text { Preferred } \\
\text { Polynomial } \\
\text { Pair Number }\end{array}$ & $\mathrm{n}$ & $\mathrm{N}$ & $\begin{array}{c}\text { Preferred } \\
\text { Polynomial (1) }\end{array}$ & $\begin{array}{c}\text { Preferred } \\
\text { Polynomial (2) }\end{array}$ \\
\hline 0 & 18 & 262143 & {$\left[\begin{array}{llllll}18 & 10 & 6 & 5 & 0\end{array}\right]$} & {$\left[\begin{array}{llll}18 & 7 & 0\end{array}\right]$} \\
\hline 1 & 5 & 31 & {$\left[\begin{array}{lll}5 & 2 & 0\end{array}\right]$} & {$\left[\begin{array}{lllll}5 & 4 & 3 & 2 & 0\end{array}\right]$} \\
\hline 2 & 6 & 63 & {$\left[\begin{array}{lll}6 & 1 & 0\end{array}\right]$} & {$\left[\begin{array}{lllll}6 & 5 & 2 & 1 & 0\end{array}\right]$} \\
\hline 3 & 7 & 127 & {$\left[\begin{array}{lll}7 & 3 & 0\end{array}\right]$} & {$\left[\begin{array}{lllll}7 & 3 & 2 & 1 & 0\end{array}\right]$} \\
\hline 4 & 9 & 511 & {$\left[\begin{array}{lll}9 & 4 & 0\end{array}\right]$} & {$\left[\begin{array}{lllll}9 & 6 & 4 & 3 & 0\end{array}\right]$} \\
\hline 5 & 10 & 1023 & {$\left[\begin{array}{lll}10 & 3 & 0\end{array}\right]$} & {$\left[\begin{array}{llll}10 & 8 & 2 & 0\end{array}\right]$} \\
\hline 6 & 11 & 2047 & 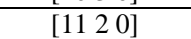 & {$\left[\begin{array}{lllll}1 & 1 & 8 & 5 & 2\end{array}\right]$} \\
\hline
\end{tabular}

\section{Effect of System Architecture}

In both GPS codes and 3GPP users' codes, the same structures of Gold codes are used. The difference is in the LFSR size or degree of polynomial. This means there might not be almost zero cross correlation between them. This suggests the possibility of intersystem interference between GPS codes and 3GPP users' codes.

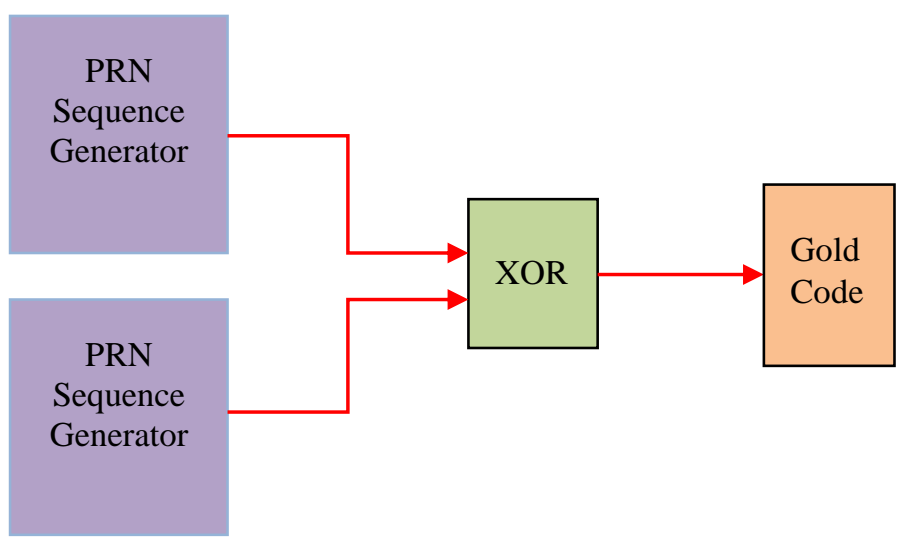

Fig. 2. Gold Code Structure

\section{E. Effect of Similarity in Polynomial Design}

It is noted that the polynomial of 3GPP is similar to the polynomial of GPS. In general, the polynomial of 3GPP code has similar structure to that of GPS polynomial.

It has been found that the polynomial pair number 5 (shown in Table 1) is equal in length to the polynomial in equation 8. This may lead to unwanted interference that, by the end, affects the system performance.

From the previous discussion, it is concluded there is a similarity between GPS and 3GPP structures i.e., using the same technique to generate the codes but with different lengths (except for preferred polynomial number 5) of polynomial or size of LFSR. From these points, we conclude 
there is a strong case that $3 \mathrm{GP}$ codes will affect the GPS code.

In this paper we do cross correlation between 32 GPS satellite codes of length 1023 chips and 3GP code using the prefer list of polynomial in 3GPP, which lead to an interference based on the results used. The analysis presented in section II should carry out to both $3 \mathrm{G}$ and $4 \mathrm{G}$ since the uplink and downlink scrambling codes are based on Gold codes [12].

\section{RESULTS}

The simulations are based on the Physical Layer of the 3GPP library in Matlab. It consists of channel coding scheme, Physical channel; mapping and transmit antenna power allocations. The following results were obtained based on each type of simulation namely; cross correlation, correlation coefficient and signal to noise ratio (SNR).

\section{A. Cross Correlation}

Cross correlation was carried out between each satellite code and satellite number 26 (PRN 26) to show the extent of correlation between the different satellites PRN codes (intra system effects). PRN 26 was arbitrarily chosen. The same results would apply if other satellites were chosen. From the output of this process the cross correlation between them approaches zero, this means zero effect interference between GPS codes. These results are shown in Fig. 3, 4, and 5. The dark blue trace illustrates the value of cross correlation between satellite number 10,13 , and 16 with satellite number 26.

On the other hand, cross correlation was carried out between each GPS code and the list of preferred polynomials in Table 1. Fig. 3, 4, and 5 show the results with their color designations.

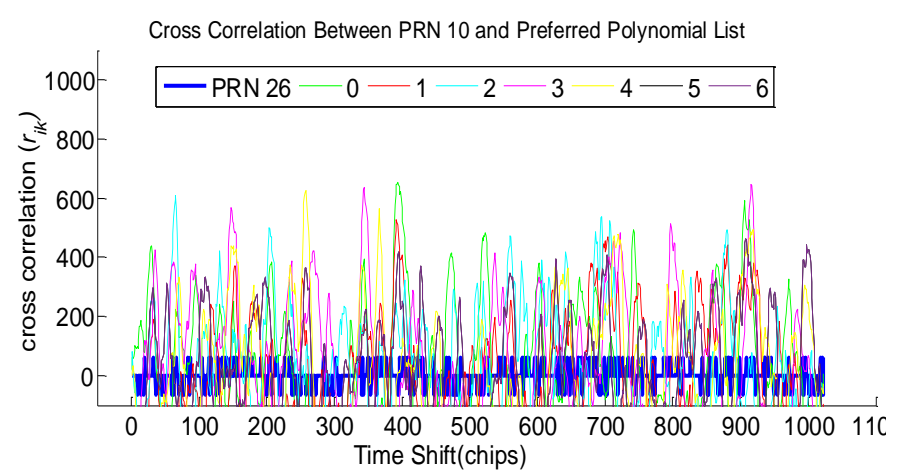

Fig. 3. Cross correlation between PRN 10 and Preferred Polynomial List (Table 1).

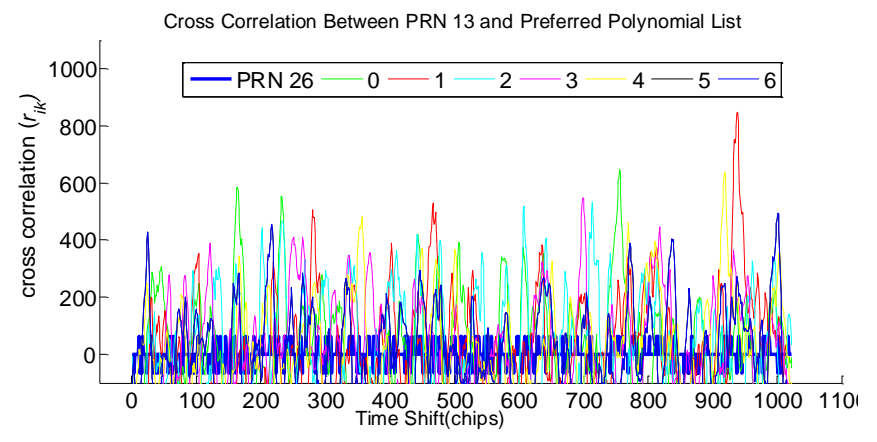

Fig. 4. Cross correlation between PRN 13 and Preferred Polynomial List (Table 1).

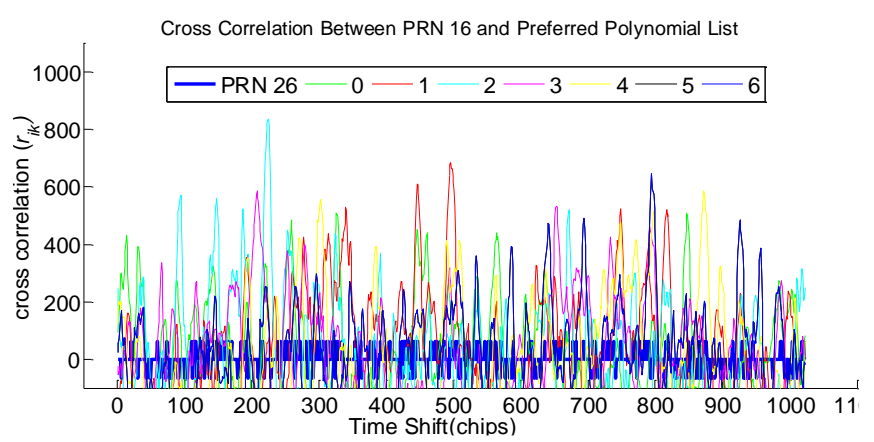

Fig. 5. Cross correlation between PRN 16 and Preferred Polynomial List (Table 1).

The three figures also show the extent of high correlation between GPS codes and 3GPP users' codes. Fig. 4 shows the significant correlation between satellite 13 and preferred polynomial number ' 1 ' (red trace), the value of cross correlation is 846.1 (approaching the maximum which is the peak value $\left.r_{k k}=1023\right)$. This large value can cause interference if the satellite interfered with attained high enough signal level that will show the drastic variations and degration in SNR.

In Fig. 5, a large value is recorded for interference between satellite number 16 and preferred polynomial number 2 (about 836.7, cyan trace), it is recommended that preferred polynomial 2 is assigned to customers in areas/regions where satellite 16 is dominant in coverage.

\section{B. Correlation Coefficient}

Appendix I shows the results for the correlation coefficient. The table has results for autocorrelation, cross correlation with PRN 26, and cross correlation with the seven preferred polynomial listed in Table 1 . These results are summarized in Fig. 6-9. It is evident that the value of correlation coefficient in the cross correlation between satellites approaches zero and this is a logical result because the interference is very limited between them. On the other hand, the value of correlation coefficient between each satellite code and other 3GPP code is large compared to value of correlation coefficient with the same satellites codes. For example, the value of correlation coefficient between satellite 11 and preferred polynomial 3 is -1 . This means there is similarity between them.

\section{Signal to Noise Ratio}

The SNR is the signal power to the noise power ratio. It gives the amount of degradation the signal suffered due to the interference. The SNR was obtained using the Software Defined Radio (SDR) receiver in [13]. It is the output of the Acquisition engine (Figure 10) of the SDR receiver where the Fast Fourier Transform (FFT) was used to process the received signal after considering the Doppler frequency to obtain the SNR. The hardware parameters of the ADC in [13] such as the sampling frequency, resolution...etc. were also taken into consideration in the SDR receiver. Figure 10 shows details of one form of GPS SDR receiver and how the SNR was obtained. 
Fig. 11 shows the results for the signal to noise (SNR) ratio. It is evident that using different polynomial codes affect the SNR especially for the satellites that record high SNR. For example, satellite number 22 could lose up to $3 \mathrm{~dB}$ in SNR ( 2 in Fig. 10) if preferred polynomial pair number 1 is used for $3 \mathrm{G} / 4 \mathrm{G}$ users' codes. Similar results could be obtained for satellites number 15 and 21 .

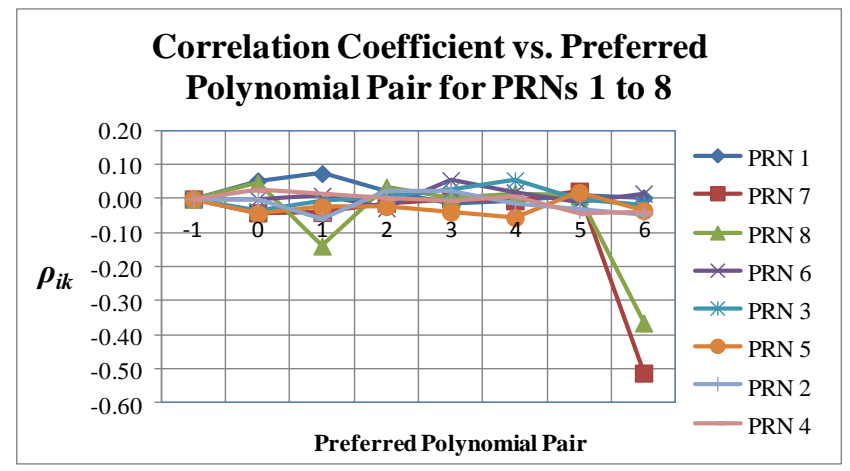

Fig. 6. Correlation Coefficient vs. Preferred Polynomial Pair for PRNs 1 to 8.

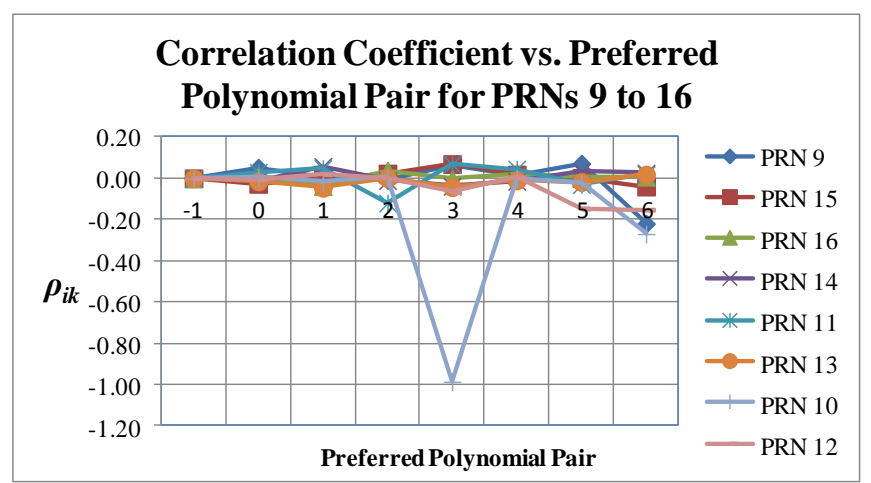

Fig. 7. Correlation Coefficient vs. Preferred Polynomial Pair for PRNs 9 to 16.

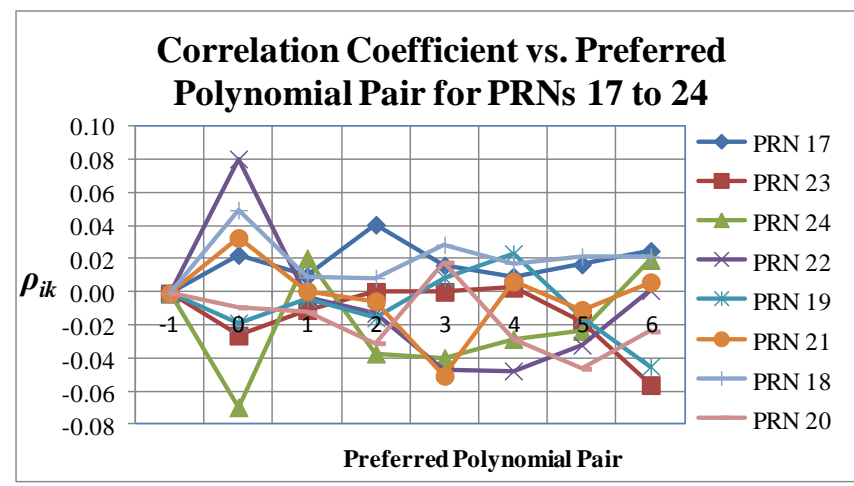

Fig. 8. Correlation Coefficient vs. Preferred Polynomial Pair for PRNs 17 to 24

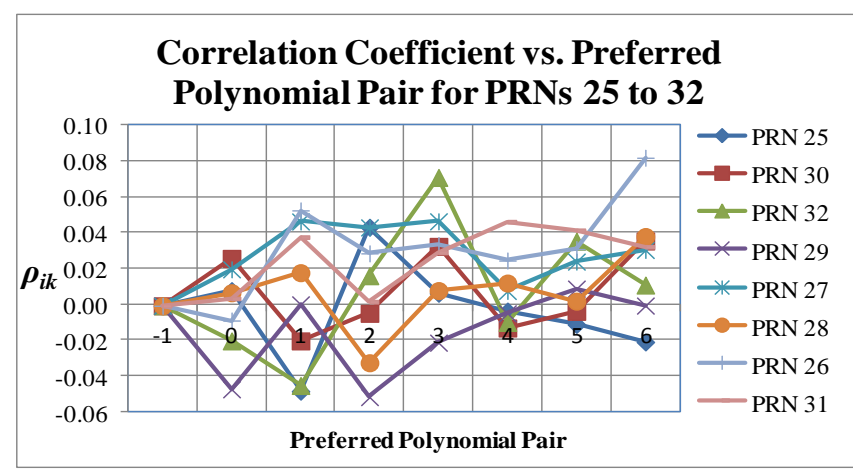

Fig. 9. Correlation Coefficient vs. Preferred Polynomial Pair for PRNs 25 to 32.

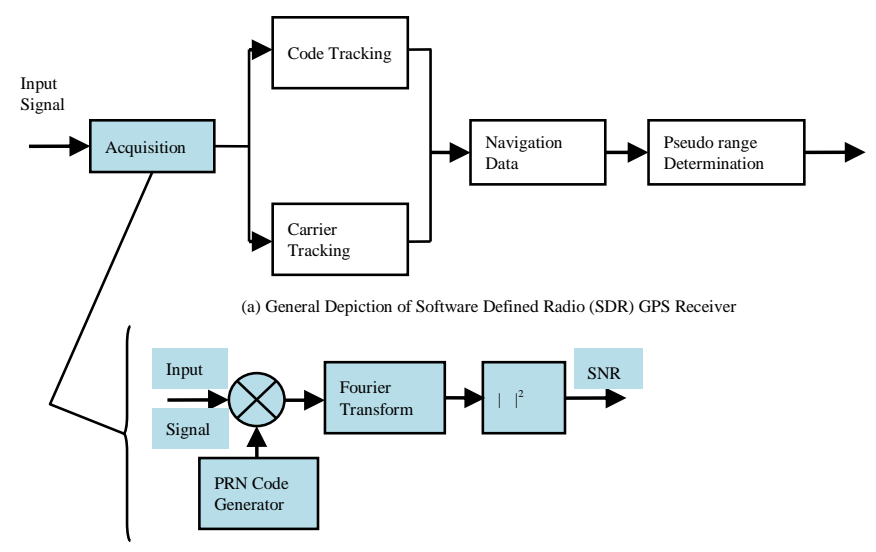

(b) Components of the Acquisition engine

Figure 10 (a) General Depiction of Software Defined Radio (SDR) GPS Receiver. (b) Components of the Acquisition Engine Showing how the SNR is obtained.

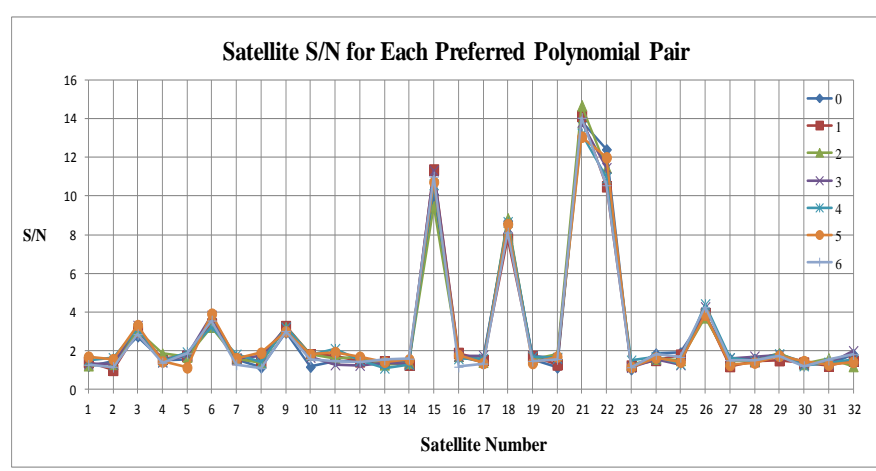

Fig. 11. Signal to noise $(\mathrm{S} / \mathrm{N})$ ratio versus satellite number for each pair of the preferred polynomial list shown in Table 1.

\section{CONCLUSION}

In this paper, the effects of 3GPP users' codes to GPS was demonstrated in the form of cross correlation, correlation coefficient and signal to noise ratio. First, the effects were demonstrated from the point of view of similarities in architecture design as well as polynomial generator design. In fact, the simulations were based on the Physical Layer of the 3GPP. The ultimate performance metric, which is the SNR, showed these effects in terms of variation in SNR values for different 3GPP polynomial pair choice. The variation could range up to $3 \mathrm{~dB}$, which is significant. One last note on this is 
that the cross correlation used here is classified as even cross correlation [1]. It is worthwhile (recommended) to further this research to include odd cross correlation and Doppler shift effects. It is also recommended that the results and comparisons made in this paper taken as guidelines when designing a $3 \mathrm{G}$ or $4 \mathrm{G}$ networks in terms of allocating users' 3GPP codes so it does not affect the GPS performance. Particularly recommended is Fig. 10.

\section{REFERENCES}

[1] S. Wallner, J. Avila-Rodriguez, G. W. Hein, and J. J. Rushanan. (2007, Sept.). Galileo E1 OS and GPS L1C Pseudo Random Noise Codes Requirements, Generation, Optimization and Comparison. Presented at 20th International Technical Meeting of the Satellite Division of The Institute of Navigation (ION GNSS 2007). [Online]. Available: https://www.ion.org/publications/abstract.cfm?articleID=7359

[2] T. AlSharabati and Y. Chen. (2010). Statistical Approach to Mitigating $3 \mathrm{G}$ Interference to GPS in 3G Handset. International Journal of Communications, Network and System Sciences. [Online] 3(09), pp. 730-736. Available: http://file.scirp.org/pdf/IJCNS20100900002 _69382778.pdf

[3] T. AlSharabati, "Effect of different interferer data rates on GPS acquisition," in NAECON, Dayton, OH, 2014, pp. 263-266.

[4] R. Prasad and T. Ojanpera, "An overview of CDMA evolution toward wideband CDMA," IEEE Comm. Surveys, vol. 1, no. 1, pp. 2-29, First Quarter 1998.

[5] I. Ahmad, "Scrambling Code Generation for WCDMA on the StarCore ${ }^{\mathrm{TM}}$ SC140/SC1400 Cores," Freescale Semiconductor, AN2254, Rev. 1, Nov 2004.

[6] P. Samundiswary and V. Kalyan. (2012, Jan.). Performance Analysis of WCDMA using Different Spreading Codes. International Journal of Computer Applications. [Online] 38(10), pp. 8-11. Available: http://citeseerx.ist.psu.edu/viewdoc/download?doi=10.1.1.259.3663\&re $\mathrm{p}=$ rep $1 \&$ type $=$ pdf

[7] H. Holma and A. Toskala, WCDMA for UMTS, Radio Access For Third Generation Mobile Communications, $3^{\text {rd }}$ ed., Chichester, England: John Wiley \& Sons, Ltd., 2004.

[8] Braun, W. R. (1997, Dec.). PN Acquisition and Tracking Performance in DS/CDMA Systems with Symbol-Length Spreading Sequences. IEEE Transactions on Communication. [Online] 45(12), pp. 15951601. Available: http://ieeexplore.ieee.org/document/650238/?reload= true\&arnumber $=650238$

[9] Y. H. Lee and S. J. Kim. (2000, Nov.). Sequence acquisition of DSCDMA systems employing Gold sequences, IEEE Transactions on Vehicular Technology. [Online] 49(6). pp. 2397-2403. Available: http://ieeexplore.ieee.org/document/901908/

[10] J. J. Benedetto, Applied and Numerical Harmonic Analysis, Boston, MA: Birkhauser, 2007.

[11] ALTERA Corp. "Gold Code Generator Reference Design." AN 295, Gold code generator reference design (2003): 1-16. [Online] Available: http://www.cs.cmu.edu/ sensing-sensors/readings/Gold_Code_Generat or-an295.pdf

[12] A. I. Johnson, "Codes in Third and Fourth Generation Networks," in $4 G$ Wireless Communication Networks: Design, Planning and Applications. Aalborg, Denmark: River Pub., 2013, pp. 152-56.

[13] K. Borre, D. Akos, N. Bertelsen, P. Rinder, and S. Jensen, A SoftwareDefined GPS and Galileo Receiver: A Single Frequency Approach. Boston: Birkhauser, 2007.

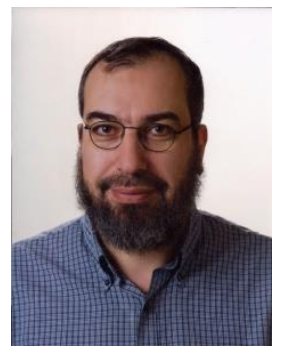

Taher Al Sharabati obtained his BSEE from St. Cloud State University in 1993, his MSEE from the University of Toledo in 1996, and his $\mathrm{PhD}$ in Electrical Engineering from the University of South Carolina in 2012.

Between his MSEE and $\mathrm{PhD}$, he worked in the industry for many years during which he worked at Motorola Inc. for about nine years. Currently, he is an assistant professor at $\mathrm{Al}$ Ahliyya Amman University in the Electronics and Communications Engineering department. 


\section{APPENDIX I}

TABLE I.1

Correlation Coefficient $\rho_{i k}$ For Satellite Auto Correlation, Cross Correlation with PRN 26 AND the Preferred Polynomial LIST.

\begin{tabular}{|c|c|c|c|c|c|c|c|c|c|}
\hline PRN & $\begin{array}{c}\text { Auto } \\
\text { Correlation }\end{array}$ & $\begin{array}{c}\text { Cross } \\
\text { Correlation }\end{array}$ & \multicolumn{7}{|c|}{ Preferred Polynomials Pair from Table I } \\
\hline 1 & 1 & $-9.784 \mathrm{e}^{-4}$ & 0 & 1 & 2 & 3 & 4 & 5 & 6 \\
\hline 2 & 1 & $-9.784 e^{-4}$ & 0.0522 & 0.0735 & 0.0211 & -0.0164 & -0.0081 & 0.009 & 0.0024 \\
\hline 3 & 1 & $-9.784 \mathrm{e}^{-4}$ & -0.0012 & -0.0615 & 0.0201 & 0.0211 & -0.0146 & -0.0328 & -0.048 \\
\hline 4 & 1 & $-9.784 e^{-4}$ & -0.0364 & -0.0077 & 0.0058 & 0.0248 & 0.0538 & -0.0031 & -0.017 \\
\hline 5 & 1 & $-9.784 \mathrm{e}^{-4}$ & 0.0244 & 0.0138 & $1.569 \mathrm{e}^{-4}$ & -0.005 & 0.0062 & -0.0433 & -0.0410 \\
\hline 6 & 1 & $-9.784 \mathrm{e}^{-4}$ & -0.0438 & -0.0247 & -0.0232 & -0.0392 & -0.0558 & 0.0182 & -0.035 \\
\hline 7 & 1 & $-9.784 \mathrm{e}^{-4}$ & -0.0018 & 0.008 & -0.028 & 0.054 & 0.0186 & -0.010 & 0.0151 \\
\hline 8 & 1 & $-9.784 \mathrm{e}^{-4}$ & -0.0416 & -0.0406 & -0.0133 & $-8.28 \mathrm{e}^{-4}$ & -0.0064 & 0.022 & -0.512 \\
\hline 9 & 1 & $-9.784 e^{-4}$ & 0.0458 & -0.139 & 0.034 & $7.24 \mathrm{e}^{-4}$ & 0.0155 & 0.0078 & -0.365 \\
\hline 10 & 1 & $-9.784 \mathrm{e}^{-4}$ & 0.0508 & 0.0025 & -0.0092 & 0.0608 & 0.0091 & 0.0704 & -0.221 \\
\hline 11 & 1 & $-9.784 \mathrm{e}^{-4}$ & 0.0034 & -0.0183 & 0.0026 & -0.987 & $1.102 \mathrm{e}^{-4}$ & -0.0246 & -0.271 \\
\hline 12 & 1 & $-9.784 \mathrm{e}^{-4}$ & 0.0286 & 0.0463 & -0.121 & 0.0669 & 0.0434 & -0.0310 & 0.0174 \\
\hline 13 & 1 & $-9.784 \mathrm{e}^{-4}$ & -0.0070 & 0.0191 & -0.0026 & -0.0679 & 0.0039 & -0.148 & -0.153 \\
\hline 14 & 1 & $-9.784 e^{-4}$ & -0.0161 & -0.0467 & $-5.539 \mathrm{e}^{-4}$ & -0.044 & -0.011 & -0.0192 & 0.0169 \\
\hline 15 & 1 & $-9.784 e^{-4}$ & -0.0123 & 0.0566 & -0.0120 & -0.0393 & -0.0152 & 0.0309 & 0.0241 \\
\hline 16 & 1 & $-9.784 \mathrm{e}^{-4}$ & -0.0278 & -0.004 & 0.022 & 0.068 & 0.017 & 0.001 & -0.0408 \\
\hline 17 & 1 & $-9.784 e^{-4}$ & 0.0146 & -0.0390 & 0.0366 & -0.0036 & 0.0193 & 0.0040 & 0.0068 \\
\hline 18 & 1 & $-9.784 e^{-4}$ & 0.0222 & 0.0094 & 0.0405 & 0.0159 & 0.0092 & 0.0165 & 0.0247 \\
\hline 19 & 1 & $-9.784 \mathrm{e}^{-4}$ & 0.0493 & 0.0088 & 0.0083 & 0.0284 & 0.0171 & 0.0213 & 0.0213 \\
\hline 20 & 1 & $-9.784 \mathrm{e}^{-4}$ & -0.0188 & -0.004 & -0.0157 & 0.0084 & 0.0231 & -0.0154 & -0.0450 \\
\hline
\end{tabular}




\begin{tabular}{|c|c|c|c|c|c|c|c|c|c|}
\hline 21 & 1 & $-9.784 \mathrm{e}^{-4}$ & -0.0094 & -0.0123 & -0.0310 & 0.0177 & -0.0288 & -0.0464 & -0.0239 \\
\hline 22 & 1 & $-9.784 \mathrm{e}^{-4}$ & 0.0325 & $4.683 \mathrm{e}^{-4}$ & -0.0056 & -0.0507 & 0.0062 & -0.0109 & 0.0058 \\
\hline 23 & 1 & $-9.784 \mathrm{e}^{-4}$ & 0.0802 & -0.0031 & -0.0130 & -0.0475 & -0.0477 & -0.0322 & $7.085 \mathrm{e}^{-4}$ \\
\hline 24 & 1 & $-9.784 \mathrm{e}^{-4}$ & -0.0264 & -0.0110 & $0-0.0607$ & 0.0083 & 0.0025 & -0.0184 & -0.0563 \\
\hline 25 & 1 & $-9.784 \mathrm{e}^{-4}$ & -0.0697 & 0.0203 & -0.0373 & -0.0399 & -0.0285 & -0.0236 & 0.0192 \\
\hline 26 & 1 & 1 & 0.0075 & -0.0487 & 0.0429 & 0.0060 & -0.0039 & -0.0114 & -0.0212 \\
\hline 27 & 1 & $-9.784 \mathrm{e}^{-4}$ & -0.0092 & 0.0523 & 0.0283 & 0.0330 & 0.0243 & 0.0307 & 0.0817 \\
\hline 28 & 1 & $-9.784 e^{-4}$ & 0.0194 & 0.0461 & 0.0428 & 0.0462 & 0.0073 & 0.0237 & 0.0302 \\
\hline 29 & 1 & $-9.784 \mathrm{e}^{-4}$ & 0.0063 & 0.0175 & -0.0328 & 0.0075 & 0.0117 & 0.0016 & 0.0377 \\
\hline 30 & 1 & $-9.784 \mathrm{e}^{-4}$ & -0.0476 & $-1.882 \mathrm{e}^{-4}$ & -0.0520 & -0.0216 & -0.0052 & 0.0086 & $-8.751 \mathrm{e}^{-4}$ \\
\hline 31 & 1 & $-9.784 \mathrm{e}^{-4}$ & 0.0255 & -0.0206 & -0.0052 & 0.0322 & -0.0134 & -0.0041 & 0.0352 \\
\hline 32 & 1 & $-9.784 e^{-4}$ & 0.0031 & 0.0374 & 0.0013 & 0.0288 & 0.0460 & 0.0412 & 0.0320 \\
\hline
\end{tabular}

